\title{
Estabilización oxidativa del aceite de Sacha inchi (Plukenetia volubilis Linneo) con suspensiones de mortiño (Vaccinium meridionale $S W$ )
}

\author{
Oxidative Stabilization of Sacha Inchi (Plukenetia Volubilis Linneo) Oil with \\ Mortiño (Vaccinium Meridionale $S W$ ) Suspensions Addition
}

K. Zapata Acosta

A. M. Piedrahita ${ }^{a}$

A. F. Alzate

F. B. Cortés ${ }^{b}$

B. A. Rojano ${ }^{\mathrm{a}, *}$

Recepción: 25-feb-15

Aceptación: 24-jun-15

\begin{abstract}
Resumen
El aceite de Sacha inchi (Plukenetia volubilis Linneo)ha sido reconocido a nivel mundial por su alto contenido de omegas; sin embargo, la presencia en él de ácidos grasos poliinsaturados hace que sea sustancialmente propenso al deterioro oxidativo. En este trabajo se evaluó el efecto de suspensiones de mortiño (Vaccinium meridionale $S W$ ) a 1000, 1500 y $2000 \mathrm{mg} \mathrm{L}^{-1}$ sobre la estabilidad oxidativa del aceite de Sacha inchi (Plukenetia volubilis Linneo)sometido a condiciones de oxidación aceleradas durante 12 horas $\left(60{ }^{\circ} \mathrm{Cy}\right.$ flujo de aire a $1700 \mathrm{~mL} \cdot \mathrm{min}^{-1}$ ). La magnitud del deterioro oxidativo fue monitoreada midiendo la formación de compuestos polares (CP), trienos conjugados (TC) y valor peróxido (VP) del aceite, en presencia y ausencia de la suspensión. Los resultados revelaron que el aditivo es eficaz en la estabilización del aceite de Sacha inchi (Plukenetia volubilis Linneo), de tal manera que concentraciones de $2000 \mathrm{mg} \mathrm{L}^{-1}$ reducen el contenido de compuestos polares, trienos conjugados y valor peróxido en un 92,3\%, 71,4\% y $47,8 \%$, respectivamente. De otro lado, se determinó el contenido de fenoles totales, flavonoides, taninos condensados y antocianinas de la suspensión de mortiño (Vaccinium meridionale $S W$ ), de modo que se caracterizara el poder antioxidante; los resultados demuestran que la suspensión es una fuente excepcional de antioxidantes naturales que serían los responsables de prevenir la oxidación del aceite de Sacha inchi (Plukenetia volubilis Linneo).
\end{abstract}

Palabras clave: antioxidantes, Mortiño (Vaccinium meridionale $S W$ ), omegas, polifenoles, Sacha inchi (Plukenetia volubilis Linneo).

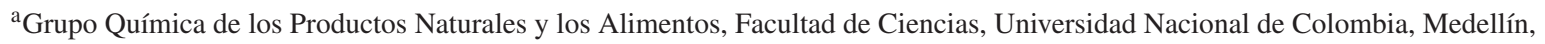
Colombia.

${ }^{\mathrm{b}}$ Grupo de Yacimientos de Hidrocarburos, Departamento de Química y Petróleos, Facultad de Minas, Universidad Nacional de Colombia, Medellín, Colombia.

*Autor de correspondencia: brojano@unal.edu.co
} 


\begin{abstract}
Sacha inchi (Plukenetia volubilis Linneo) oil has been recognized worldwide for its high Omega content. However, the presence of polyunsaturated fatty acids make the oil substantially susceptible to oxidative deterioration. In this study, the effect of mortiño (Vaccinium meridionale SW) suspensions at 1000, 1500 and $2000 \mathrm{mg} \mathrm{L}^{-1}$ on the oxidative stability of Sacha inchi (Plukenetia volubilis Linneo),oil under accelerated oxidation conditions for 12 hours $\left(60^{\circ} \mathrm{Cand} 1700 \mathrm{~mL}\right.$.min- 1 air flow) was evaluated. The extent of oxidative damage was monitored by measuring the formation of polar compounds (CP), conjugated trienes (TC) and peroxide value (PV) of the oil in the presence and absence of the suspension. The results revealed that the additive is effective in stabilizing Sacha inchi (Plukenetia volubilis Linneo) oil, so that concentrations of $2000 \mathrm{mg} \mathrm{L}^{-1}$ reduced the content of polar compounds, conjugated trienes and peroxide value in $92.3 \%$, $71.4 \%$ and $47.8 \%$ respectively. On the other hand, the content of total phenols, flavonoids, condensed tannins and anthocyanins in the mortiño (Vaccinium meridionale $S W$ ), suspension was determined, showing that the suspension is a unique source of natural antioxidants that would be responsible for preventing the oxidation of Sacha inchi (Plukenetia volubilis Linneo) oil.
\end{abstract}

Key words: Antioxidants, Mortiño (Vaccinium Meridionale SW), Oil, Oxidation, Polyphenols, Prevention.

\section{Introducción}

Sacha inchi (Plukenetia volubilis Linneo) es una planta oleaginosa nativa de la región amazónica del Perú, con semillas de, aproximadamente, $2 \mathrm{~cm}$ de diámetro, que se componen básicamente, de $33 \%$ de proteína y $54 \%$ de lípidos [1]. En la fracción lipídica, más del $48 \%$ corresponde a ácidos grasos Omega 3; aproximadamente el $37 \%$, a ácidos grasos Omega 6, y el $8 \%$, a ácidos grasos Omega 9 [2]; es decir, junto con el alto contenido de Omegas, el aceite tiene un contenido excepcionalmente bajo de ácidos grasos saturados (6\%), lo que es de suma importancia desde el punto de vista nutracéutico. Entre los beneficios asociados al consumo de Omegas se hallan: la disminución de los niveles de triacilgliceridos, la disminución del riesgo a sufrir arritmias, disminución de la placa arterioesclerótica, regulación de la tensión arterial, entre otros [3]. A su vez, el aceite de Sacha inchi (Plukenetia volubilis Linneo) es rico en vitaminas liposolubles como la A y la E. Todas estas ventajas han hecho del aceite de Sacha inchi (Plukenetia volubilis Linneo) un producto reconocido internacionalmente por sus propiedades nutracéuticas. No obstante, es un producto de poco uso en la agroindustria, sobre todo por su susceptibilidad al daño oxidativo [4].

El alto contenido de ácidos grasos poliinsaturados ( $>90 \%)$ hacen que el aceite de Sacha inchi (Plukenetia volubilis Linneo) sea propenso al deterioro oxidativo [5]; este fenómeno, también conocido como rancidez oxidativa, genera productos primarios de oxidación como peróxidos e hidroperóxidos, y productos secundarios, en su mayoría volátiles, como aldehídos, cetonas y ácidos; o no volátiles, como dímeros, trímeros y polímeros [6]. Los productos de oxidación alteran significativamente las propiedades nutracéuticas y sensoriales del aceite, generando olores y sabores indeseables y provocan la pérdida de valor nutricional, debido a la oxidación de omegas y a la descomposición de las vitaminas A, E y D, lo que lleva a una depreciación del producto y a un rechazo del consumidor.

La adición de antioxidantes a los aceites se hace con el objetivo de retardar los procesos de rancidez oxidativa, disminuir la posibilidad de generación de compuestos tóxicos, evitar la decoloración de los pigmentos y disminuir la pérdida de valor nutricional causada por la degradación de los ácidos grasos esenciales y por la destrucción de las vitaminas A, E y D [7-8]; además, si los antioxidantes son de origen natural, refuerzan la actividad de los sistemas antioxidantes endógenos del consumidor, aportando una protección extra ante el estrés oxidativo.

En la actualidad, los antioxidantes sintéticos son los más utilizados en la industria de los alimentos y demás; sin embargo, existe preocupación respecto a su seguridad. El butilhidroxianisol (BHA) y el butilhidroxitolueno (BHT) han exhibido efectos adversos a la salud, como el aumento del colesterol, hepatomegalia e inducción de cáncer hepático, entre otros. Por ello, se ha dirigido el interés hacia el encuentro y adición de antioxidantes naturales, ya que se 
consideran más seguros, inocuos y, además, actúan a bajas concentraciones [9-10].

Los extractos vegetales son, entonces, una alternativa, ya que tienen una variedad de compuestos fenólicos capaces de atrapar especies reactivas del oxígeno. Especialmente, el género Vaccinium tiene cerca de 400 especies, y sus frutos han atraído el interés de muchos investigadores alrededor del mundo, debido al alto contenido de compuestos antioxidantes, tales como ácido cinámico, flavonoles, antocianinas y antocianidinas [11-13], superior a los valores reportados para frutos de ingesta frecuente en Colombia, como la mora, la piña, el melón, la guayaba, el mango, la fresa, la granadilla y la guanábana, entre otros.

Muchos estudios dan cuenta de la capacidad de antioxidantes sintéticos y naturales para inhibir de la peroxidación lipídica de matrices alimenticias [1418]; sin embargo, a la fecha solo un estudio ha utilizado el Vaccinium meridionale $S W$ para tal propósito; este trabajo fue realizado por Montoya et al. (2009), y en él se evaluó el efecto de los extractos antociánicos del mortiño (Vaccinium meridionale $S W$ ), sobre la peroxidación lipídica de aceite de maíz, analizando la evolución de dienos conjugados (DC), el valor de peróxidos (VP) y la formación de sustancias reactivas con el ácido 2-tiobarbitúrico (TBARS), como indicadores de la oxidación; aunque los resultados fueron positivos cuando se utilizaban concentraciones del extracto superiores a $500 \mu \mathrm{mg} \mathrm{L}^{-1}$, estos no fueron significativos, debido a que la reducción en la producción de DC, VP y TBARS fue inferior, en todos los casos, al $10 \%$.

Dados los antecedentes, y considerando la excepcional actividad antioxidante del mortiño (Vaccinium meridionale $S W$ ), este trabajo tiene como objetivo evaluar la capacidad de los extractos del fruto para inhibir la formación de productos de oxidación como hidroperóxidos, trienos conjugados y compuestos polares del aceite de Sacha inchi (Plukenetia volubilis Linneo) sometido a condiciones aceleradas de oxidación, con el fin de ampliar y profundizar en el conocimiento científico sobre el mortiño (Vaccinium meridionale $S W$ )como antioxidante multipropósito, y el aceite de Sacha inchi (Plukenetia volubilis Linneo) como alimento funcional.

Este trabajo busca intervenir y mejorar la situación problema propuesta, y formula un posible prototipo para la industria alimenticia; concretamente, desea generar alternativas naturales para la preservación de las propiedades fisicoquímicas del aceite de Sacha inchi (Plukenetia volubilis Linneo), debido a que es una fuente promisoria de metabolitos nutracéuticos.

\section{Materiales y métodos}

\section{Perfil lipídico del aceite de Sacha inchi (Plukene- tia volubilis Linneo)}

Para determinar el perfil de ácidos grasos del aceite de Sacha inchi (Plukenetia volubilis Linneo) se empleó un cromatógrafo de gases CG 6890N Agilent, acoplado a un detector selectivo MS 5973N Agilent y equipado con un inyector Split/splitless. La temperatura del inyector fue $300 \pm 1{ }^{\circ} \mathrm{C}$. Las muestras, previamente derivatizadas, para facilitar su detección como metil ésteres (FAME), fueron inyectadas automáticamente en el modo splitless. Se empleó una columna HP-5 ms. (5\% fenilmetil siloxano) de $30 \mathrm{~m}, 0,25 \mathrm{~mm}$, con un espesor de película de $0,25 \mu \mathrm{m}$ y una temperatura máxima de $325 \pm{ }^{\circ} \mathrm{C}$. Para identificar el tipo de ácido graso presente se empleó la base de datos NIST 98 [19].

\section{Obtención de la pulpa de mortiño (Vaccinium me- ridionale $S W$ )}

Fueron lavados $200 \mathrm{~g}$ de fruta y desinfectados con hipoclorito de sodio $100 \mathrm{ppm}$; luego, fueron despulpados a través de Ultra-Turrax Brand: IKAWERK $^{\circledR}$ durante $10 \mathrm{~min}$ a $10000 \mathrm{rpm}$, hasta obtener una pasta uniforme. La pulpa fresca se almacenó en bolsas de polietileno con cierre hermético y se congeló a $-20{ }^{\circ} \mathrm{C}$ hasta su uso.

\section{Liofilización de la pulpa de mortiño (Vaccinium meridionale $S W$ )}

La liofilización fue llevada a cabo en un liofilizador de mesa marca Labotec, referencia 12FD. Para ello, $100 \mathrm{~g}$ de pulpa fueron depositados en platos de aluminio de $18 \mathrm{~cm}$ de diámetro y $2 \mathrm{~cm}$ de alto. Los platos fueron ubicados dentro del liofilizador sobre su condensador para llevarlos a temperatura de congelación de $-40{ }^{\circ} \mathrm{C}$. La temperatura de la placa de calentamiento avanzó desde -40 hasta $+40{ }^{\circ} \mathrm{C}$. El vacío de la cámara fue de $15 \mathrm{~Pa}$ y el tiempo de permanencia de la muestra fue aproximadamente 8 horas. Se obtuvieron liofilizados con humedades finales aproximadas del $10 \%$. 


\section{Preparación de las suspensiones de mortiño (Vac- cinium meridionale $S W$ )}

Para la incorporación del liofilizado de mortiño (Vaccinium meridionale $S W$ ) al aceite de Sacha inchi (Plukenetia volubilis Linneo) se preparó previamente una suspensión; para esto, aproximadamente 10 $\mathrm{g}$ de liofilizado fueron mezclados con $250 \mathrm{~mL}$ de etanol (solvente de extracción), $2 \mathrm{~mL}$ de polisorbato (emulsificante) y $50 \mathrm{~mL}$ de propilenglicol (dispersante). La mezcla fue homogeneizada con Ultra-Turrax Brand: IKA-WERK ${ }^{\circledR}$ durante 5 min a $10000 \mathrm{rpm}$, y sometida a agitación magnética durante 1 hora a $90{ }^{\circ} \mathrm{C}$, para favorecer la extracción de antocianinas; ensayos previos señalan que la extracción de antocianinas del Vaccinium meridionale $S W$ se potencia con el uso de energía, siempre y cuando la temperatura de extracción esté entre 80 y $90{ }^{\circ} \mathrm{C}$ [20]. Finalmente, se retiró el exceso de etanol por evaporación al vacío y hasta obtener un producto viscoso.

\section{Caracterización de las suspensiones de mortiño} (Vaccinium meridionale $S W$ )

Se determinó la distribución y el tamaño de partícula a través del Analizador "NanoPlus DLS Nano Particle Size and Zeta Potential", y, además, la viscosidad de la suspensión elaborada, haciendo uso del equipo "Viscosímetro Fungilab ALPHA L".

Determinación del contenido polifenólico en suspensiones de mortiño (Vaccinium meridionale $S W$ )

Para la evaluación del contenido fenólico en las suspensiones, estas fueron diluidas en etanol hasta lograr concentraciones que permitieran el análisis experimental correspondiente.

Fenoles totales: la determinación de fenoles se realizó por el método colorimétrico de FolinCiocalteu, descrito por Singleton y Rossi [21], con algunas modificaciones. Volúmenes de $50 \mu \mathrm{L}$ de la suspensión coloidal fueron adicionados a $125 \mu \mathrm{L}$ del reactivo de Folin y $400 \mu \mathrm{L}$ de carbonato de Sodio $7,1 \%$, ajustando con agua destilada hasta $1000 \mu \mathrm{L}$. La lectura espectrofotométrica se realizó a $760 \mathrm{~nm}$, y se comparó con la curva patrón usando como estándar ácido gálico (fenol). Los resultados fueron expresados como mg de ácido gálico equivalentes por gramo de liofilizado en suspensión.
Taninos condensados: este método colorimétrico se fundamenta en la reacción de los taninos condensados con vainillina bajo condiciones ácidas, como lo describen Hagerman y Butler [22]. Un volumen de $230 \mu \mathrm{L}$ de la suspensión coloidal fue adicionado a $670 \mu \mathrm{L}$ de una solución de vainillina recién preparada $(1 \mathrm{~g}$ en $100 \mathrm{~mL})$ en ácido sulfúrico al $70 \%$. La mezcla se incubó a $20{ }^{\circ} \mathrm{C}$ durante $15 \mathrm{~min}$; se realizó la lectura espectrofotométrica a $500 \mathrm{~nm}$ y se comparó con la curva patrón usando como estándar (+)-catequina. Los resultados fueron expresados como mg de Catequina equivalentes por gramo de liofilizado en suspensión.

Flavonoides totales: la determinación de flavonoides se realizó siguiendo el método colorimétrico descrito por Marinova y col. [23], con algunas modificaciones. Volúmenes de $100 \mu \mathrm{L}$ de la suspensión coloidal fueron mezclados con $30 \mu \mathrm{L}$ de $\mathrm{NaNO}_{2}$ al $5 \%, 30 \mu \mathrm{L}$ de $\mathrm{AlCl}_{3}$ al $10 \%, 200 \mu \mathrm{L}$ de $\mathrm{NaOH}$ a $1 \mathrm{M}$ y ajustados con agua destilada hasta un volumen final de 1,000 $\mu \mathrm{L}$; se realizó la lectura espectrofotométrica a $510 \mathrm{~nm}$ y se comparó con la curva patrón usando como estándar (+)-catequina. Los resultados fueron expresados como mg de Catequina equivalente por gramo de liofilizado en suspensión.

Antocianinas totales: las antocianinas totales se determinaron mediante el método diferencial de $\mathrm{pH}$ descrito por Gaviria y col. [24], con algunas modificaciones. Volúmenes de $100 \mu \mathrm{L}$ de la suspensión coloidal fueron mezclados $900 \mu \mathrm{L}$ de buffer de $\mathrm{pH} 1,0$ y 4,5 , y se leyeron las absorbancias a $530 \mathrm{~nm}$ y 700 nm; para el cálculo del contenido de antocianinas, expresadas como equivalentes de cianidin-3-glucósido, se usó la expresión $\mathrm{A}=\left[(\mathrm{A} 530-\mathrm{A} 700)_{\mathrm{pH} 1,0}-(\mathrm{A} 530-\right.$ A700 $)_{\mathrm{pH} 4.5}$ ] y el coeficiente de extinción molar de 26,900. Los resultados se expresaron como mg equivalentes de cianidin-3-glucósido por gramo de liofilizado en suspensión.

Estabilización del aceite: la incorporación del extracto vegetal al aceite se realizó según el método descrito por Sultana y col. [25]. La suspensión de mortiño (Vaccinium meridionale $S W$ ) fue agregada al aceite de Sacha inchi (Plukenetia volubilis Linneo), logrando concentraciones finales de 1,000, 1,500 y 2,000 $\mathrm{ppm}$. Las muestras de aceite fueron agitadas en Vortex durante $10 \mathrm{~min}$, hasta obtener dispersiones uniformes. Los aceites tratados y la muestra control (aceite sin antioxidante) fueron sometidos durante 
12 horas a condiciones aceleradas de oxidación, es decir, $60{ }^{\circ} \mathrm{C}$ y un flujo de aire constante de 1,700 $\mathrm{mL} \cdot \mathrm{min}^{-1}$. Todos los aceites fueron preparados por triplicado. Muestras de aceite fueron tomadas cada hora hasta 12 horas. El nivel de deterioro oxidativo fue evaluado por la medición del valor peróxido (VP), trienos conjugados (TC) y compuesto polares (CP).

Determinación de la estabilidad oxidativa del aceite de Sacha inchi (Plukenetia volubilis Linneo)

Determinación de compuestos polares (CP): para monitorear el contenido de compuestos polares (CP) se utilizó el dispositivo TESTO 270 - TESTEADOR DE ACEITES DE FRITURA (Testo Inc., Germany), como lo sugiere Wei-any col. [26]. El dispositivo es sumergido dentro de la muestra y es agitado suavemente durante $20 \mathrm{~s}$ para permitir la adecuada distribución de la muestra. Este rápido método detecta la constante dieléctrica del aceite otorgada por la presencia de compuestos polares. Esta constante es convertida a \% CP basado en fórmulas establecidas por los fabricantes.

Determinación de trienos conjugados (TC): el contenido de TC se determinó siguiendo el método descrito por Zuta y col. [27], con algunas modificaciones: Aproximadamente $20 \mathrm{mg}$ de cada aceite fueron pesados y mezclados con $2,000 \mu \mathrm{L}$ de ciclohexano; la mezcla se agitó en vortex durante $10 \mathrm{~s}$, y luego se determinó la absorbancia a $260 \mathrm{~nm}$. Para los resultados se utilizó la ley de Beer, teniendo en cuenta el coeficiente de extinción molar para los trienos conjugados $\left(2,525 \mathrm{M}^{-1} \mathrm{~cm}^{-1}\right)$. Los valores de trienos conjugados (TC) se expresan como $\mu \mathrm{mol}$ TC por cada $g$ de aceite.

Determinación del valor peróxido (VP): el valor de peróxidos se determinó mediante el método descrito por Shanta y Decker [28], con algunas modificaciones; este método se fundamenta en la capacidad de los peróxidos lipídicos de oxidar el $\mathrm{Fe}^{+2}$ hasta $\mathrm{Fe}^{+3}$. Aproximadamente $20 \mathrm{mg}$ de aceite fueron pesados y mezclados con $3,5 \mathrm{~mL}$ de una solución cloroformo:metanol (7:3); luego, a $1 \mathrm{~mL}$ de la mezcla se le adicionaron $50 \mu \mathrm{L}$ de una solución de sulfato de hierro en cloruro de bario $\left(\mathrm{Fe}^{+2}\right), 50 \mu \mathrm{L}$ de tiocianato de amonio al $30 \%$ y $900 \mu \mathrm{L}$ de cloroformo. La reacción se dejó incubar durante 10 min en la oscuridad, a temperatura ambiente; posteriormente, se determinó la absorbancia a $500 \mathrm{~nm}$. Los resultados se expresan como miliequivalentes de oxígeno por cada $\mathrm{kg}$ de aceite, mediante el uso de una curva estándar construida usando $\mathrm{Fe}^{+3}$ y tiocianato de amonio como patrón.

Para expresar los resultados como equivalentes de oxígeno se utilizó (1):

Miliequivalentes de Oxígeno $=$

$$
A /(55,84 \times 0,114672 \times B) \times(100 / C)
$$

donde $A$ es igual a Absorbancia de la muestra a $500 \mathrm{~nm} ; B$ es la concentración inicial de la muestra (aceite en cloroformo: metanol), y $C$ es el porcentaje de dilución de la muestra (en caso de ser necesario).

Análisis estadístico: dos muestras de cada aceite tratamiento/control fueron sometidas a oxidación y analizadas individualmente por triplicado; los resultados son reportados como la media \pm desviación estándar. El análisis de varianza fue de una vía, con un nivel de significancia del 95\% ( $p<$ $0,05)$, usando el programa Statgraphics Plus versión 5,0 (StatisticalGraphics Corp., Rockville, MD). Las gráficas fueron obtenidas a través del programa OriginPro 8,0.

\section{Resultados y discusión}

Perfil lipídico del aceite de Sacha inchi (Plukenetia volubilis Linneo)

La velocidad de degradación de los lípidos no solo depende de la exposición a temperatura y oxígeno, también, de la composición del aceite; por ejemplo, el grado de insaturación es un factor que afecta directamente la cinética de las reacciones de degradación [29-32]. Aceites con un alto contenido de ácidos grasos insaturados, especialmente poliinsaturados, son más susceptibles a la oxidación, por lo tanto, el tipo y concentración de los ácidos grasos presentes en el aceite es un parámetro importante en la caracterización de aceites vegetales con diferentes fines [33].

La tabla 1 muestra el porcentaje de ácidos grasos de aceite de Sacha inchi (Plukenetia volubilis Linneo). Los resultados revelaron que tan solo el $13 \%$ del contenido de ácidos grasos totales corresponden a Ácidos Grasos Saturados (AGS), y aproximadamente el $85 \%$ son Ácidos Grasos Poliinsaturados (AGP); de estos últimos, el 83\% corresponde a Ácido Linolénico-Omega 3, y el 2\%, a Ácido Linoléico- 
Omega 6. La composición lipídica del Aceite de Sacha inchi (Plukenetia volubilis Linneo) es comparable con los reportes existentes para el aceite de linaza; sin embargo, composicionalmente es muy diferente a los resultados hallados en aceites vegetales usados en la industria alimenticia para fritura. Se sabe que los aceites de fritura deben cumplir ciertas características, como tener un alto contenido de Ácidos Grasos Saturados y ser ricos en Ácido Oleico; esta composición les permite soportar mejor que los aceites muy insaturados las altas temperaturas del proceso de fritura y la oxidación [34].

Tabla 1. Contenido de ácidos grasos del Aceite de Sacha inchi (Plukenetia volubilis Linneo).

\begin{tabular}{lcc}
\hline Nombre común & $\begin{array}{c}\text { Carbonos: } \\
\text { insaturaciones }\end{array}$ & $\begin{array}{c}\text { Porcentaje relativo } \\
\text { composicional }\end{array}$ \\
\hline Ácido palmítico & $16: 0$ & $7,79 \%$ \\
Ácido palmitoleico & $16: 1$ & $0,24 \%$ \\
Ácido esteárico & $18: 0$ & $4,89 \%$ \\
Ácido linoléico (Omega 6) & $18: 2^{\Delta 9,12}$ & $1,66 \%$ \\
Ácido linolénico (Omega 3) & $18: 3^{\Delta 9,12,15}$ & $82,71 \%$ \\
\hline
\end{tabular}

Si bien el aceite de Sacha inchi (Plukenetia volubilis Linneo) no sería una opción como aceite de fritura, podría usarse en preparaciones en fresco, similar al aceite de oliva. Los resultados obtenidos indicaron que el aceite de Sacha inchi (Plukenetia volubilis Linneo) es fuente excepcional de AGP, que, según evidencia científica existente, son indispensables para el cuerpo humano, pues realizan funciones como el mantenimiento de membranas celulares y la producción de prostaglandinas (reguladores de procesos corporales, entre ellos la inflamación y la coagulación sanguínea); además se ha demostrado experimentalmente que el consumo de grandes cantidades de omega-3 aumenta considerablemente el tiempo de coagulación de la sangre, lo cual explica por qué en comunidades que consumen muchos alimentos con omega-3 [35], la incidencia de enfermedades cardiovasculares es sumamente baja [36]. En un estudio hecho por Garmendia y cols. [37] se evalúo el efecto de la ingesta de aceite de Sacha inchi (Plukenetia volubilis Linneo) sobre el perfil lipídico de pacientes con hipercolesterolemia, para quienes se determinaron los valores sanguíneos de colesterol total (CT), colesterol de baja densidad (LDL), colesterol de alta densidad (HDL), triglicéridos (Tg), glucosa e insulina; los resultados revelaron una disminución de las concentraciones de CT, LDL y Tg con dosis de $5 \mathrm{~mL}$ de aceite, al mismo tiempo que una elevación muy importante del colesterol de alta densidad (HDL).

Estos resultados, sumados a los aquí reportados, dan cuenta de los beneficios en salud que presenta este tipo de alimento; sin embargo, se deben utilizar estrategias alimentarias, como la que se plantea en esta investigación, que no solo permiten la conservación de las propiedades organolépticas y nutracéuticas del aceite, evitando su oxidación, sino que lo conviertan en un alimento funcional.

\section{Caracterización de la suspensión de mortiño (Vaccinium meridionale $S W$ )}

La suspensiones obtenidas son de tipo químico (no coloidales), debido a que las partículas de la fase dispersa son visibles a nivel macroscópico, presentando tamaños superiores a $1 \mu \mathrm{m}$, específicamente, entre 2,9 y $8,3 \mu \mathrm{m}$, con tamaño promedio de $5,6 \mu \mathrm{m}$. La distribución del tamaño de partículas sigue un comportamiento gaussiano unimodal, como se aprecia en la figura 1, lo que indica que es probable que la mayoría de partículas presenten tamaños similares al tamaño medio 5,6 $\mu \mathrm{m}$.

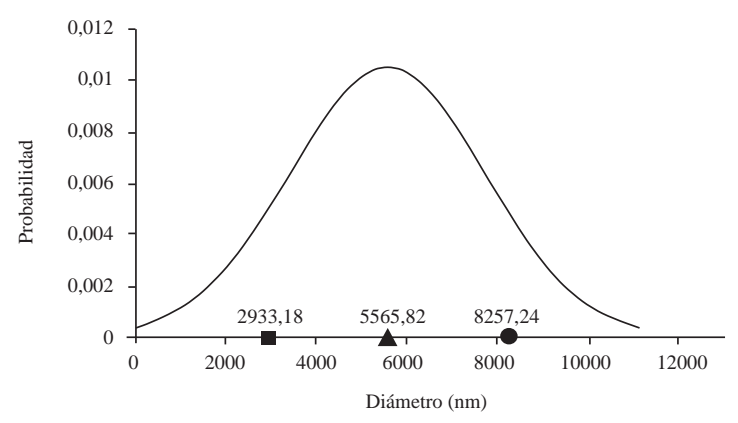

Figura 1. Distribución del tamaño de partícula de las suspensiones de mortiño (Vaccinium meridionale $S W$ )

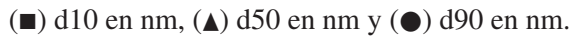

Los resultados señalan, además, que el $90 \%$ de las partículas presentan un tamaño igual o menor a $8,3 \mu \mathrm{m}$ y le otorgan una viscosidad a la suspensión de $28,15 \mathrm{Cp}$, medida a $26{ }^{\circ} \mathrm{C}$.

\section{Contenido Polifenólico en suspensiones de morti- ño (Vaccinium meridionale $S W$ )}

El contenido de fenoles, taninos condesados, flavonoides y antocianinas de la suspensión de mortiño (Vaccinium meridionale $S W$ ) se presenta en la tabla 2; los resultados fueron expresados por gramo de liofilizado. 
En un estudio hecho por Zapata y col. [38] se determinó el contenido fenólico y la capacidad antioxidante total de frutas y verduras de mayor consumo en Colombia; los resultados fueron usados para clasificar los productos en tres grupos: bajo $(<100)$, medio (100-500) y alto contenido fenólico ( $>500$ ), y son expresados como mg de Fenoles Totales por cada $100 \mathrm{~g}$ muestra liofilizada. De acuerdo con esta información, la suspensión elaborada se considera un producto con alto contenido fenólico, y los resultados son similares a los reportados para alimentos ricos en polifenoles, como la granadilla, la guayaba, el mortiño, la fresa, la mora y la curuba.

Tabla 2. Contenido de fenoles, taninos condesados, flavonoides y antocianinas de la suspensión de mortiño (Vaccinium meridionale $\mathrm{Sw}$ ).

\begin{tabular}{|c|c|c|c|}
\hline les & sados & totales & \\
\hline $\begin{array}{l}\text { mg Ácido } \\
\text { Gálico equi- } \\
\text { valente por } \\
100 \mathrm{~g} \text { de } \\
\text { liofilizado }\end{array}$ & $\begin{array}{l}\text { mg Catequi- } \\
\text { na equivalen- } \\
\text { te por } 100 \\
\text { g de liofiliza- } \\
\text { do }\end{array}$ & $\begin{array}{l}\text { mg Catequi- } \\
\text { na equivalen- } \\
\text { te por } 100 \\
\text { g de liofiliza- } \\
\text { do }\end{array}$ & $\begin{array}{l}\text { in-3- } \\
\text { ido } \\
0 \mathrm{~g} \text { de } \\
\text { ado }\end{array}$ \\
\hline 2,15 & 00 & 6,36 & $\begin{array}{l}46 \\
9,3\end{array}$ \\
\hline
\end{tabular}

Por otro lado, el contenido de antocianinas totales fue superior $(463 \pm 9,3)$ a los valores reportados para frutos de otras especies del género. Prior y col. [39] reportaron contenidos de antocianinas totales de blueberry (Vaccinium corymbosum) entre 92 y 235; 60-187 para el Rabbiteye blueberry (Vaccinium ashei) y 290-300 para Lowbush blueberry (Vaccinium angustifolium) [39]. Frutos como la mora, el açaí y la uva han sido reconocidos por su alto contenido de antocianinas, sin embargo, ninguno supera el contenido antociánico reportado para las especies de Vaccinium [40].

Los taninos condensados, proantocianidinas y procianidinas son polímeros formados por unidades de flavan-3-ol (catequina, epicatequina, antocianinas) y se caracterizan por conferirle astringencia a los frutos; son, además, uno de los principales metabolitos antioxidantes de productos como el té y el cacao, cuyo contenido puede llegar hasta el $35 \%$ de los polifenoles totales; en este estudio se determinó que los taninos condensados constituyen el 27,52\% de los fenoles hallados en la suspensión.
Los flavonoides constituyen el grupo de compuestos fenólicos más diverso y ampliamente distribuido en las plantas; su estructura básica (flaván) consta de dos grupos fenilos unidos por un puente de tres carbonos que forma un anillo heterocíclico oxigenado; esta combinación C6-C3-C6 es llamada con frecuencia esqueleto difenil-propano, determinante en su reactividad química [41]. Los flavonoides tienen un alto poder atrapador de radicales libres; además, tienen actividad antiinflamatoria, antiviral, antibacterial y coadyuvante en la disminución del riesgo a padecer enfermedades cardiovasculares [42]. Para la suspensión, el contenido de flavonoides fue similar al reportado para frutas de consumo frecuente como la pera, la manzana, la mora, la frambuesa y la fresa, entre otras.

Estos metabolitos secundarios con estructuras fenólicas determinan la actividad antioxidante de las matrices debido a su capacidad para donar electrones y protones y neutralizar especies reactivas de oxígeno [43].

\section{Estabilidad oxidativa del aceite de Sacha inchi (Plukenetia volubilis Linneo)}

La susceptibilidad de los lípidos a la oxidación es a menudo evaluada determinando la concentración de productos de oxidación intermedios y finales [44]. En esta investigación se determinaron productos totales e intermedios de oxidación mediante la aplicación de técnicas como \% Compuestos Polares y Trienos conjugados/Valor peróxido, respectivamente.

Los Compuestos Polares (CP), según la Norma Técnica Colombiana (NTC 5225), incluyen sustancias presentes en grasas no sometidas a altas temperaturas, tales como monoglicéridos, diglicéridos, ácidos grasos libres y otros, al igual que los productos de oxidación final (aldehídos y cetonas) originados en su mayoría durante el calentamiento del aceite [45].

La figura 2 muestra la evolución en la formación de Compuestos Polares del aceite de Sacha inchi (Plukenetia volubilis Linneo) sometido a condiciones aceleradas de oxidación con y sin suspensiones de mortiño (Vaccinium meridionale $S W$ ). Durante las primeras 6 horas, el contenido de $\mathrm{CP}$ es constante y no se observa un efecto protector de la suspensión; sin embargo, después de este tiempo la producción 
de Polares se incrementa y el efecto del incorporado se hace visible: la adición de 1500 y $2000 \mathrm{mg} \mathrm{L}^{-1}$ de suspensiones al aceite redujo la velocidad de producción de CP en el 88,0 y 92,3\%, respectivamente. Durante todo el experimento, los valores de CP en presencia de las suspensiones (concentración $=1500$ $\mathrm{mg} \mathrm{L}^{-1}$ ) fueron inferiores a $24 \%$, lo que sugiere que la adición de suplementos para aumentar la vida útil del aceite de Sacha inchi (Plukenetia volubilis Linneo) es ideal.

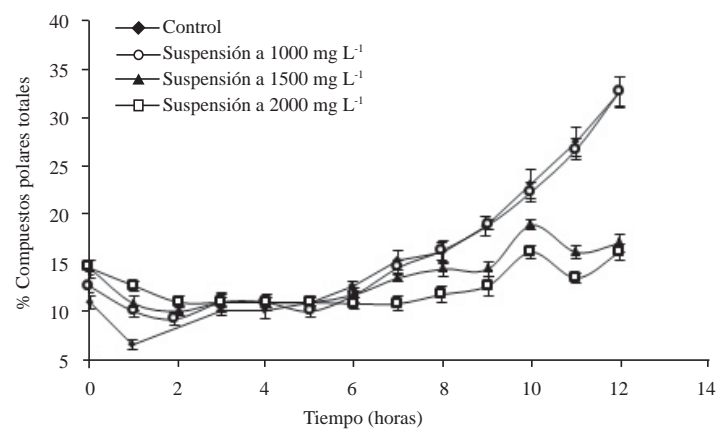

Figura 2. Evolución en la formación de Compuestos Polares (CP) del aceite de Sacha inchi (Plukenetia volubilis Linneo) adicionado de suspensiones de mortiño (Vaccinium meridionale $S W$ ) ( $) 0 \mathrm{mg} \mathrm{L}^{-1},(\bigcirc) 1,000$ $\mathrm{mg} \mathrm{L}^{-1},(\boldsymbol{\Lambda}) 1,500 \mathrm{mg} \mathrm{L}^{-1}, \mathrm{y}(\square) 2,000 \mathrm{mg} \mathrm{L}^{-1}$.

El CP se mide como indicador de deterioro oxidativo y criterio de desecho de aceites y grasas, y la regulación es exigente al prohibir aceites con valores superiores al $27 \%$ de CP.

Los aceites con valores altos de CP presentan modificaciones de tipos sensoriales y nutricionales [4647]; además, algunos investigadores han reportado que cuando se suministran altas cantidades de $\mathrm{CP}$ a animales de experimentación, estos presentan retraso en el crecimiento fetal, hipertrofia o hiperplasia hepática, hígado graso, úlceras gástricas y lesiones tisulares en corazón y riñón [48-49].

Los Trienos Conjugados son productos intermediarios del proceso de oxidación; se forman por el reordenamiento de los dobles enlaces de los ácidos grasos insaturados que se han convertido en radicales lipídicos [50]. Este experimento reveló que existe un incremento del contenido de trienos conjugados a medida de que transcurre el tiempo de uso; además, las muestras oleosas con suspensiones de mortiño (Vaccinium meridionale $S W$ ) mostraron niveles más bajos de trienos conjugados, comparadas con el control (figura 3); el uso de 1,000, 1,500 y 2,000 mg
$\mathrm{L}^{-1}$ de suspensión inhibió en 9,5, 61,9 y 71,4\% la formación de trienos conjugados, respectivamente.

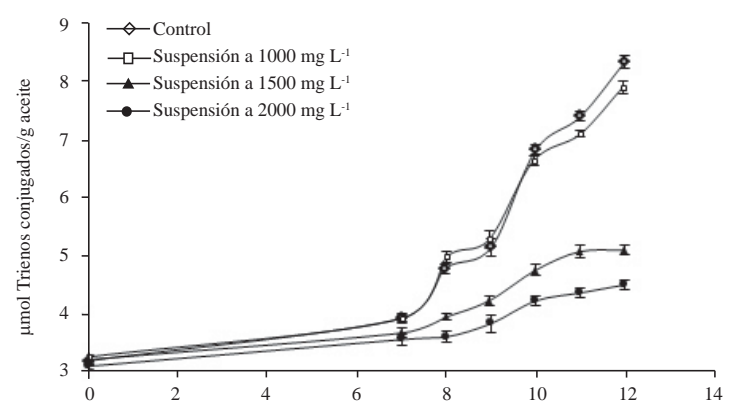

Figura 3. Evolución en la formación de Trienos Conjugados (TC) del aceite de Sacha inchi (Plukenetia volubilis Linneo) adicionado de suspensiones de mortiño (Vaccinium meridionale $S W)(\diamond) 0 \mathrm{mg} \mathrm{L}^{-1},(\square) 1,000$ $\mathrm{mg} \mathrm{L}^{-1}$, (ム) $1,500 \mathrm{mg} \mathrm{L}^{-1}$ y (•) $2,000 \mathrm{mg} \mathrm{L}^{-1}$.

Estos resultados permiten predecir que los metabolitos antioxidantes presentes en la suspensión de mortiño (Vaccinium meridionale $S W$ )actúan tanto en las etapas finales de la peroxidación lipídica como durante las etapas iníciales e intermedias.

El valor peróxido es un parámetro de oxidación primaria ampliamente utilizado para definir la calidad oxidativa de un aceite. Los radicales libres y otras especies reactivas son capaces de extraer un átomo de hidrógeno de un ácido graso poliinsaturado, generando un radical lipídico (R·) que puede reaccionar con $\mathrm{O}_{2}$, dando lugar a un radical peroxilo $\left(\mathrm{ROO}^{-}\right)$. Los radicales peroxilo contribuyen a la propagación de las reacciones en cadena de peroxidación lipídica, sustrayendo átomos de hidrógeno de otros ácidos grasos insaturados cercanos, generando así nuevos radicales lipídicos (R·) e hidroperóxidos lipídicos $(\mathrm{ROOH})$; estos últimos se convierten en buenos indicadores del estado oxidativo de un aceite; este valor, medido en miliequivalentes de oxígeno por $\mathrm{kg}$ de aceite presenta un valor limitante para el consumo igual a 20; valores menores son característicos de aceites no oxidados, y valores por encima indican que el proceso de oxidación ha comenzado [51].

En este experimento, el VP inicial para el aceite de Sacha inchi (Plukenetia volubilis Linneo) fue 2,8, correspondiente a un aceite no oxidado, y, como es de esperarse, el contenido incrementó con el tiempo de oxidación (figura 4). Las muestras con suspensiones mostraron niveles más bajos de VP, comparadas 
con el control, y se evidencia que existe una relación directa entre la concentración de aditivo y la inhibición en la aparición de peróxidos, por ejemplo, concentraciones de $1,000,1,500$ y $2,000 \mathrm{mg} \mathrm{L}^{-1}$ de suspensión en el aceite inhibieron en 10,9, 34,8 y 47,8\% la formación de peróxidos.

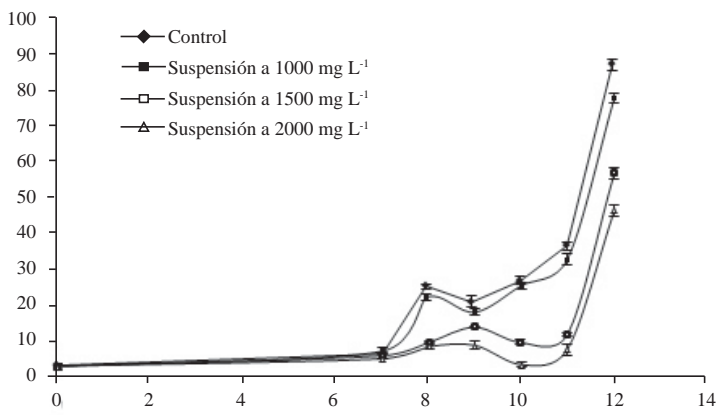

Figura 4. Evolución de la formación de Peróxidos (VP) del aceite de Sacha inchi (Plukenetia volubilis Linneo) adicionado de suspensiones de mortiño (Vaccinium meridionale $S W$ ) $(\downarrow) 0 \mathrm{mg} \mathrm{L}^{-1}$, (ロ) $1,000 \mathrm{mg} \mathrm{L}^{-1},(\square)$ $1,500 \mathrm{mg} \mathrm{L}^{-1}$ y $(\triangle) 2,000 \mathrm{mg} \mathrm{L}^{-1}$.

Si bien no existen estudios sobre suplementación de aceites con suspensiones de mortiño (Vaccinium meridionale $S W$ ), varios autores han evaluado el poder antioxidante de extractos vegetales sobre la peroxidación lipídica de matrices oleosas y han ofrecido nuevas alternativas en la estabilización de aceites. Iqbal y Bhanger [52] determinaron la capacidad antioxidante de extractos de ajo en aceite de girasol bajo condiciones aceleradas; Budryn y col. [53] determinaron el poder de los extractos de café para inhibir la peroxidación lipídica del aceite de girasol; Sultana y col. [25] obtuvieron resultados similares al estabilizar aceite de maíz con extractos de residuos de frutas agroalimentarios; así, un sinnúmero de autores concluyen sobre la efectividad de los extractos naturales sobre la prevención de fenómenos oxidativos en aceites [54-56].

\section{Conclusión}

Los resultados de esta investigación revelaron que, composicionalmente, el aceite de Sacha inchi (Plukenetia volubilis Linneo) no es una alternativa como aceite de fritura; sin embargo, podría consumirse en fresco, con múltiples ventajas para el consumidor, como el aporte de Omegas, pero el alto contenido de ácidos grasos poliinsaturados hace de este alimento un material susceptible al deterioro oxidati- vo; por ello, esta investigación propone alternativas naturales para incrementar la vida en anaquel de este producto, $\mathrm{y}$, además, presenta ventajas adicionales como el refuerzo de los sistemas antioxidantes endógenos del consumidor, aportando una protección extra ante el estrés oxidativo.

Los resultados fueron satisfactorios, pues la suspensión de mortiño (Vaccinium meridionale $S W$ ), arrojo porcentajes de inhibición en la formación de productos de oxidación, como compuestos polares, trienos conjugados y peróxidos, superiores al $50 \%$, cuando se utilizan concentraciones entre 1500 y $2000 \mathrm{ppm}$.

\section{Referencias}

[1] L. Follegatti-Romeroa, C. Piantinoa, R. Grimaldib y F. Cabrala, "Supercritical $\mathrm{CO}_{2}$ extraction of omega-3 rich oil from SachaInchi (Plukenetiavolubilis L.) seeds", J. Supercrit Fluid, vol. 49, pp. 323-329, Jul. 2009.

[2] ProFound. Biocomercio Perú. 19 de 03 de 2010. Recuperado de: http://www.biocomercioperu.org

[3] Pulevasalud. 01 de 10 de 2008. Recuperado de: http://www.pulevasalud.com

[4] M. D. Guillén, A. Ruiz, N. Cabo, R. Chirinos y G. Pascual, "Characterization of sacha inchi (Plukenetia volubilis L.) oil by FTIR spectroscopy and ${ }^{1} \mathrm{H}$ NMR Comparison with linseed oil”, J. AOCS, vol. 80, pp. 755-762, Jul. 2003.

[5] N. Maurer, B. Hatta-Sakoda, G. PascualChagman y L. Rodríguez-Saona, "Characterization and authentication of a novel vegetable source of omega-3 fatty acids, sachainchi (Plukenetiavolubilis L.) oil", Food Chem., vol. 134, pp. 1173-1180, Sep. 2012.

[6] V. Coello-Frías "Efecto de la adición de Ácido Ascórbico y Butil Hidroxitolueno (BHT) en la oxidación enzimática y rancidez oxidativa de pasta de aguacate (Persea americana) variedades Hass y Bacon". Tesis de maestría, Fac. de Ciencias e Ing. de Alimentos, Universidad Técnica de Ambato, Ecuador, 2015. 
[7] A. Sánchez, "Aceites y grasas vegetales como ingrediente funcional en productos cárnicos", Nacameh., vol. 5, pp. 108-118, 2011.

[8] B. Rojano, C. Gaviria, M. Gil, J. Saez, G. Schinella y H. Tournier, "Actividad antioxidante del isoespintanol en diferentes medios", Vitae, vol. 15 pp. 173-181, 2008.

[9] N. Niæiforoviæ, V. Mihailoviæ, P. Maskoviæ, S. Solujiæ, A. Stojkoviæ, D. Pavloviæ Muratspahiæ D. "Antioxidant activity of selected plant species; potential new sources of natural antioxidants", Food and Chemical Toxicology, vol. 48, pp. 3125-3130, nov. 2010.

[10] D. Krishnaiah, R. Sarbatly y R. Nithyanandam, "A review of the antioxidant potential of medicinal plant species", Food and Bioproducts Processing, vol. 89, pp. 217-233, jul, 2011.

[11] G. A. Garzón, C. E. Narváez, K. M. Riedl y S. J. Schwartz, "Chemical composition, anthocyanins, non-anthocyanin phenolics and antioxidant activity of wild bilberry (Vaccinium meridionale Swartz) from Colombia", Food Chemistry, vol. 122, pp. 980-986, Mar 2010.

[12] Y. E. Lopera, J. Fantinelli, L. F. González. B. Rojano, J. L. Ríos, G. Schinella y S. Mosca, "Antioxidant Activity and Cardioprotective Effect of a Nonalcoholic Extract of Vaccinium meridionale Swartz during IschemiaReperfusion in Rats", Hindawi., vol. 2013, pp. 1-10, 2013.

[13] F. P. Prencipe, R. Bruni, A. Guerrini, D. Rossi, S. Benvenuti y F. Pellati, "Metabolite profiling of polyphenols in Vaccinium berries and determination of their chemopreventive properties", Journal of Pharmaceutical and Biomedical Analysis, vol. 89, pp. 257-267, 2014.

[14] B. A. Rojano, C. A. Gaviria y J. A. Sáez, "Determinación de la actividad antioxidante en un modelo de peroxidación lipídica de mantequilla inhibida por el isoespintanol", VITAE, vol. 15, n. ${ }^{\circ}$, pp. 212-218, 2008.

[15] L. Kulanthaivel, P. Srinivasan, V. Shanmugam y B. Maruthaiveeran, "Therapeutic efficacy of kaempferol against AFB1 induced experimental hepatocarcinogenesis with reference to lipid peroxidation, antioxidants and biotransformation enzymes", Biomedicine $\mathcal{F}$ Preventive $\mathrm{Nu}$ trition, vol. 2, pp. 252-259, 2012.

[16] G, Azofeifa, S. Quesada, A. M. Pérez, F, Vaillant y A. Michel, "Pasteurization of blackberry juice preserves polyphenol-dependent inhibition for lipid peroxidation and intracellular radicals", Journal of Food Composition and Analysis, vol. 42, pp. 56-62, 2015.

[17] V. K. Bajpai, A. Sharma, S. Hong Kim y K. Baek, "Chemical Composition, Antioxidant, Lipid Peroxidation Inhibition and Free Radical Scavenging Activities of Microwave Extracted Essential Oil from Allium sativum", Journal of Essential Oil Bearing Plants, vol. 18, no. 2, pp. 300-313, Jul 2015.

[18] A. Afify, H. El-Beltagi1, A. Aly y A. E ElAnsary, "Antioxidant enzyme activities and lipid peroxidation as biomarker for potato tuber stored by two essential oils from Caraway and Clove and its main component carvone and eugenol". Asian Pacific Journal of Tropical Biomedicine, vol. 2, no. 2, pp. 772-780, Ago 2012.

[19] A. Zapata-Luján, A. Cogollo-Pacheco y B. Rojano, "Potencial nutraceútico del aceite de la almendra de choibá o almendro de montaña (Dipteryx oleífera Benth.) Nutraceutical potential of Choibá almond oil or mountain almond (Dipteryxoleifera Benth.)", "Rev Cubana Plant Med, vol. 18, pp. 368-380, Abr. 2013.

[20] S. C. Paladino. "Actividad antioxidante de los compuestos fenólicos contenidos en las semillas de la vid (Vitis vinifera 1.)". Tesis de Magister en Alimentos, posgrado Regional Cooperativo en Alimentos, Universidades Nacionales de Cuyo, La Rioja, San Juan y San Luis. Facultad de Ciencias Agrarias, pp. 1-100.

[21] V. Singleton, J. Rossi, "Colorimetry of total phenolics with phosphomolybdicphosphotungstic acid reagents", Am J EnolViticult, vol. 16, pp. 144-158, 1965.

[22] A. Hagerman, L. Butler, "Choosing appropriate methods and standards for assaying tannin", $J$. ChemEcol, vol. 15, pp. 1795-1810, Jun. 1989. 
[23] D. Marinova, F. Ribarova y M. Atanassova, "Total phenolics and total flavonoids in bulgarian fruits and vegetables", Journal of the University of Chemical Technology and Metallurgy, vol. 40, pp. 255-260, Jul. 2005.

[24] C. Gaviria, O. Cifuentes, C. Monsalve y B. Rojano, "Actividad antioxidante de extractosmetanólicos de Attaleabutyracea", Sci Tech, vol. 33, pp. 297-299, May. 2007.

[25] B. Sultana, F. Anwar, A. Rafique y S. ShahidChatha, "Antioxidant potential of extracts from different agro wastes: Stabilization of corn oil", GrasasAceites, vol. 59, pp. 205-217, Sep. 2008.

[26] C. Wei-an, C. Chihwei, C. Wei-chih, H. Chaokai y K. Meng-I, “Total polar compounds and acid values of repeatedly used frying oils measured by standard and rapid methods", J Food Drug Anal, vol. 21, pp. 58-65, Oct. 2013.

[27] P. Zuta, B. Simpson, X. Zhao y L. Leclerc, "The effect of $\alpha$-tocopherol on the oxidation of mackerel oil", Food Chem, vol. 100, pp. 800807, Dic. 2007.

[28] N. Shanta, E. Decker, "Rapid, sensitive, ironbased spectrophotometric methods for determination of peroxide values of food lipids", $J$ AOAC, vol 77, pp. 421-424, Mar. 1994.

[29] M.D. Guillén y N, Cabo, "Fourier Transform Infrared Spectra Data versus Peroxide and Anisidine Values to Determine Oxidative Stability of Edible Oils", Food Chemistry, vol. 77, pp. 503-510, 2002. DOI: http://dx.doi.org/10.1016/S03088146(01)00371-5

[30] E. Choe y D. Min. "Chemistry and Reactions of Reactive Oxygen Species in Foods. Critical Reviews". Food Science and Nutrition, vol. 46, pp. 1-22, 2006. DOI: http://dx.doi.org/10.1080/10408390500455474

[31] P.J. García-Moreno, R. Pérez-Gálvez, A. Guadix, y E. M. Guadix, "Influence of the Parameters of the Rancimat Test on the Determination of the Oxidative Stability Index of Cod Liver Oil", Food Science and Technology, vol. 51, pp. 303-308, 2013. DOI: http://dx.doi.org/10.1016/j.lwt.2012.11.002
[32] A. Martínez-Yusta y M. D. Guillén, "DeepFrying. A Study of the Influence of the Frying Medium and the Food Nature, on the Lipidic Composition of the Fried Food, Using $1 \mathrm{H} \mathrm{Nu}-$ clear Magnetic Resonance", Food Research International, vol. 62, pp. 998-1007, 2014. DOI: http://dx.doi.org/10.1016/j.foodres.2014.05.015

[33] A, Fernández-Iglesias, H. Quesada, S. Díaz, D, Pajuelo, C, Bladé, L, Arola, M. J. Salvadó y M. Mulero, "Combination of Grape Seed Proanthocyanidin Extract and Docosahexaenoic Acid-Rich Oil Increases the Hepatic Detoxification by GST Mediated GSH Conjugation in a Lipidic Postprandial State", Food Chemistry, vol. 165, pp. 14-20, 2014. DOI: http://dx.doi.org/10.1016/j.foodchem.2014.05.057

[34] N. Izquierdo, L. Aguirrezábal, F. Andrade, y V. Pereyra, "Night Temperature Affects Fatty Acid Composition in Sunflower Oil Depending on the Hybrid and the Phenological Stage", Field Crops Research, vol. 77, pp. 115-126, 2002. DOI: http://dx.doi.org/10.1016/S03784290(02)00060-6

[35] E. López-Huertas, "Health effects of oleic acid and long chain omega 3 fatty acids (EPA and DHA) enriched milks A review of intervention studies", Pharmacol Res., vol. 61, pp. 200-207, Mar. 2010.

[36] K. Dhakal, K. Jung, J. Chae, J. Shannon y J. Lee, "Variation of unsaturated fatty acids in soybean sprout of high oleic acid accessions", Food Chem, vol. 164, pp. 70-73, Dic. 2014.

[37] F. Garmendia, R. Pando y G. Ronceros, "Efecto del aceite de sacha inchi (PlukenetiaVolúbilis L) sobre el perfil lipídico en pacientes con hiperlipoproteinemia", RevPeruMedExp Salud Publica, vol. 28, pp. 628-632, Dic. 2011.

[38] S, Zapata, A. M. Piedrahita y B. Rojano, "Capacidad atrapadora de radicales oxígeno (ORAC) $\mathrm{y}$ fenoles totales de frutas y hortalizas de Colombia", Perspect Nutr Humana, vol. 16, pp. 25-36, 2014.

[39] C. Gaviria, C. Ochoa, N. Sánchez, C. Medina, M, Lobo, P. Galeano, A. Mosquera, A. Tamayo, 
Y. Lopera y B. Rojano, "Actividad antioxidante e inhibición de la peroxidación lipídica de extractos de frutos de mortiño (Vaccinium meridionale SW)", Boletín Latinoamericano y del Caribe de Plantas Medicinales y Aromáticas, vol. 8, no. 6, pp. 519-528, 2009.

[40] E. M. Kuskoski, A. García, M. Teresa y R. Fett, "Frutos tropicais silvestres e polpas de frutas congeladas: atividade antioxidante, polifenóis e antocianinas". Ciência Rural, vol. 36, no. 4, jul-ago, 2006.

[41] C. Manach, A. Scalbert, C. Morand, C. Rémésy y L. Jiménez, "Polyphenols: food sources and bioavailability", American Journal of Clinical Nutrition, vol. 79, no. 5, pp. 727-747, 2004.

[42] E.J.R., Middleton, C. Kandaswami y T. Theoharidesl, "The effects of plant flavonoids on mammalian cells: Implications for inflammation, heart disease and cancer", Pharmacological Reviews, vol. 52, no. 4, pp. 673-751, 2000.

[43] P. Galeano-García, "Efecto en la peroxidación lipídica del isoespintanol y análogos”, Tesis de maestría, Fac. de Ciencias, Escuela de Química, Universidad Nacional de Colombia, sede Medellín, Colombia, 2009.

[44] Pinchuk, I. and Lichtenberg, D. "Analysis of the Kinetics of Lipid Peroxidation in Terms of Characteristic TimePoints", Chemistry and Physics of Lipids, no. 178, pp. 63-76, 2014. DOI: http://dx.doi.org/10.1016/j.chemphyslip.2013.12.001

[45] NTC 5225, Grasas y aceites animales y vegetales: determinación del contenido de compuestos polares, 2003.

[46] J. Marcano, Y. La Rosaa y N. Salinasa, "Influencia del proceso de fritura en profundidad sobre el perfil lipídico de la grasa contenida en patatas tipo "french", empleando oleina de palma”. Grasas y aceites, vol. 61, n. ${ }^{\circ} 1$, pp. 29-24, Enero 2010.

[47] A.C. Suaterna Hurtado, "La fritura de los alimentos: el aceite de fritura", Perspectivas en Nutrición Humana, vol. 11, pp. 39-53, Mayo 2009.
[48] C.M. Ramírez Botero, B. D. Gómez Ramírez, A. C. Suaterna Hurtado, J. P. Martínez Galán, L. M. Cardona Zuleta, B.A. Rojano, "Contenido de compuestos polares totales en aceites de cocina previo uso más vendidos en Medellín (Colombia)", Perspectivas en Nutrición Humana, vol. 14, pp. 59-69, Mayo 2012.

[49] M. Ruiz-Méndes, M. Dobarganes, Oil refining [online]. España: Instituto de la Grasa, 2011 Disponible en: http://lipidlibrary.aocs.org/frying/c-refining/ index.htm.

[50] M. Guichardant, P. Chen, M. Liu, E. Véricel, M. Lagarde, "Conjugated trienes in fatty acids and oxygenated derivatives", Chemistry and Physics of Lipids, vol. 163, pp. S32, Aug. 2010.

[51] M. Martínez, "Extracción y caracterización de aceite de nuez (Juglans regia 1.): influencia del cultivar y de factores tecnológicos sobre su composición y estabilidad oxidativa", Tesis Doctoral, Fac. de Ciencias Exactas, Físicas y Naturales, Universidad Nacional de Córdoba, 2010.

[52] S. Iqbal, M. Bhanger, "Stabilization of sun flower oil by garlic extract during accelerated storage", Food Chem, vol. 100, pp. 246-254, Sep. 2007.

[53] G. Budryn, E. Nebesny y D. Żyżelewicz, "Oxidative stability of lard and sunflower oil supplemented with coffee extracts under storage conditions", GrasasAceites, vol. 62, Abr. 2011.

[54] R. A. Trindadea, J. Mancini-Filhob, A.L.C.H. Villavicencio, "Natural antioxidants protecting irradiated beef burgers from lipid oxidation", Food Science and Technology, vol. 43, pp. 98104, Jan. 2010.

[55] F. Anjum, F. Anwar, A. Jamil y M. Iqbal, "Microwave roasting effects on the physicochemical composition and oxidative stability of sunflower seed oil", J AOCS, vol. 83, pp. 777-784, Sep. 2006. 
[56] A. Lante, T. Nardi , F. Zocca, A. Giacomini y V. Corich, "Evaluation of Red Chicory Extract as a Natural Antioxidant by Pure Lipid Oxidation and Yeast Oxidative Stress Response as Model
Systems", J. Agric. Food Chem., vol. 59, pp. 5318-5324, Abr. 2011. 